\title{
Metabolic profiling of liver in C57BL/6 mice with DSS- induced inflammatory bowel disease by untargeted metabolomics analysis
}

\section{Zhongquan Xin}

South China Agricultural University

Zhenya Zhai

Newhope Liuhe co.Ltd, Chendu.China

Hongrong Long

South China Agricultural University

Fan Zhang

South China Agricultural University

Xiaojun Ni

South China Agricultural University

Jinping Deng

South China Agricultural University

Lunzhao Yi

Kunming University of Science and Technology

Baichuan Deng ( $\sim$ dengbaichuan@scau.edu.cn )

South China Agricultural University https://orcid.org/0000-0002-0713-1463

\section{Research article}

Keywords: inflammatory bowel disease, liver, UPLC-HRMS, metabolomics, C57BL/6 mice

Posted Date: October 31st, 2019

DOI: https://doi.org/10.21203/rs.2.16664/v1

License: (c) (i) This work is licensed under a Creative Commons Attribution 4.0 International License. Read Full License 


\section{Abstract}

Background: Inflammatory bowel disease (IBD) is a systemic disease that frequently causes liver damage. However, the metabolic mechanism of liver disorder induced by IBD is still unclear. This study aimed to revile metabolic profiles of liver in mice which induced inflammatory bowel disease by dextran sulfate sodium (DSS) and treated by cecropin A and gentamicin .

Methods: In this study, IBD mold mice were established, C57BL/ 6 mice were given water containing $2.5 \%$ dextran sulfate sodium (DSS) for 5 days. Subsequently, the mice were treated via intraperitoneally injected with saline, $15 \mathrm{mg} / \mathrm{kg}$ cecropin A, $5 \mathrm{mg} / \mathrm{kg}$ gentamicin for 3 days, respectively. The liver in IBD mold mice or treated by cecropin A and gentamicin, were performed metabolomics analysis through UPLC-Orbitrap-MS/MS. Database integration analysis software was used to identify metabolites of liver. Multivariate analysis, including principal component analysis (PCA), hierarchical cluster analysis (HCA) and orthogonal partial least-squares discriminant analysis (OPLS-DA). Furthermore, metabolic pathway analysis was performed using MetaboAnalyst 4.0.

Results: A total of 133 metabolites were identified in liver of mice and 20 key metabolites were considered as potential biomarkers were identified between the control and IBD group. There are 4 key metabolic pathways include bile acid metabolism, arachidonic acid metabolism, amino acid and protein metabolism, and steroid hormone biosynthesis in liver was disturbed by IBD, and we also found that those pathways were reversed when mice were treated by cecropin A and gentamicin.

Conclusion: The results indicated that those differential metabolites and metabolic pathways changed in liver were likely to be caused by IBD. The further study shall be carried out to explore the mechanism of liver disorder induced by IBD.

\section{Background}

Inflammatory bowel disease (IBD) is a complex chronic disease of the gastrointestinal tract, including Crohn's disease (CD) and ulcerative colitis (UC) [1]. It may be caused by an interplay between a dysfunctional host immune response and environmental triggers (including the gut microbiome)[2]. IBD as a systemic disorder has been associated with many extraintestinal manifestations (EIMs). Almost all organ systems are involved, including the musculoskeletal,

dermatologic, renal, hepatobiliary, pulmonary and ocular systems. [3] As the main metabolic organ, liver interacts with the intestinal tract directly through the hepatic hilum and bile secretion system. Therefore, liver disorder is frequently observed in IBD and along with a varied hepatobiliary disease such as fatty liver, autoimmune hepatitis, and cirrhosis[4]. It has been estimated that about $5 \%$ of the patients with IBD developed serious liver disease[5].

Previous studies have shown that the pathophysiology and treatment of various liver diseases may be influenced by the nature and structure of the gut microbiome[6]. Removing microbes from the gut or adding them to alter the structure of colonial bacteria can cause liver disease[7, 8]. Infection with Gram-negative bacteria such as Escherichia coli (E. coli) and Salmonella is the primary cause of IBD $[1,9]$. Antibiotics are commonly used to relieve IBD through the elimination of harmful bacteria and reducing inflammation[10,11]. Recently, we found that cecropin A can also alleviate IBD and enhance the abundance of gut microflora[12]. In spite of the scientific advances in pathology study on liver disorder associated with IBD, its metabolic mechanism is still obscure.

Metabolites are small-molecular-weight intermediate and end products (endogenous or exogenous) of genes and proteins in a living organism, and all the metabolites generated by a system in an organism (e.g., cell, organ, tissue) constitute a metabolome. Metabolomics is a hot technologic and powerful high-throughput platform to identify and quantify metabolites in the organism and to find the relationship between metabolites and physiological /pathological changes[13]. In addition, metabolomics could reveal potential biomarkers in the pathological process and identify 
metabolic pathways and genes associated with the measured metabolites. The ultra-high performance liquid chromatography coupled with high-resolution mass spectrometry (UPLC-HRMS) is one of the most efficient and robust methods of generating metabolite profiles for biologic samples, because of its high throughput, resolution, and sensitivity $[14,15]$. However, accurately identifying metabolites from MS data has been generally represented as a challenge, especially in untargeted analysis [16]. Thus, there are many free or commercialized MS analysis tools, such as XCMS[17], MZmine [18], and Compound Discoverer 2.1 has been developed and committed to processing MS data acquired on liquid chromatography mass spectrometry (LC-MS) or gas chromatography mass spectrometry (GC-MS). Additionally, chemometric methods have been applied in metabolomics to processing and extracting information from high-throughput datasets[19]. Through the efficient analytical instruments coupled with effective data analysis methods, metabolomics provides a possibility to elucidate biochemical pathways of liver metabolism and offers an opportunity for discovering novel biomarkers in the process of IBD.

In this study, we aimed to develop a profile of liver metabolism of DSS induced IBD model in C57BL/6 mice. We performed metabolomics analysis on four groups: the control group, the DSS group, the CA group (Cecropin A treatment) and the GA (Gentamicin treatment). Metabolomics method based on UPLC-HRMS with database integration analysis software has been used to identify metabolites of liver of four groups. Through multivariate statistical, the potential biomarkers of liver in IBD were screened out, and relative pathways were explained. The flow chart of this study was shown in supplementary information Figure S1. We hope to explain the effect of IBD on liver metabolism at the molecular level with the aid of metabolomics.

\section{Methods}

\section{Ethics Statement】}

The experimental design and procedures in this study were reviewed and approved by the Animal Care and Use Committee of the Institute of Subtropical Agriculture, Chinese Academy of Science (No.ISA-2018-035). The National Centre for the Replacement, Refinement and Reduction of Animals in Research (NC3Rs) handling guidelines were also followed within this study. A An animal research: reporting of in-vivo experiments (ARRIVE) guidelines checklist for working with animals can be found in Additional file 1.

\section{Chemicals and reagents}

Acetonitrile (LC-MS grade) and methanol (LC-MS grade) were purchased from Merck, formic acid (LC-MS grade) and dextran sulfate sodium (DSS) were purchased from J\&K Scientific Limited. Ultra-pure water was prepared from ELGA system (RIGHTLEDER International Holding Group Limited).

\section{Animals}

Thirty-six C57BL/ 6 mice at a 3-week-old were purchased from Guangdong Medical Laboratory Animal Center. The mice were housed for 3 per cage and placed under the standard conditions (temperature, $24 \pm 1^{\circ} \mathrm{C}$; lighting cycle, $12 \mathrm{~h}: 12 \mathrm{~h}$ light/dark; 7:00 -19:00 for light) and had free access to food and drinking water.

\section{DSS-induced IBD model in mice and treatment}

The detail processes of establishing the IBD model have shown in our published research[12]. Briefly, mice were housed until 6-7 weeks of age and divided into four groups ( $\mathrm{n}=9$ mice per group). The IBD model was induced by giving water 
containing 2.5\% DSS for 5 days. Then, the mice were treated via intraperitoneally injected with saline (DSS group), 15 $\mathrm{mg} / \mathrm{kg}$ cecropin A (CA group), $5 \mathrm{mg} / \mathrm{kg}$ gentamicin (GA group) for 3 days, respectively. The control group received drinking water and were intraperitoneally injected with saline. All mice were sacrificed by $\mathrm{CO} 2$ on the eighth day, and the liver, distal ileum, and colon were collected and stored in $4 \%$ neutral polyformaldehyde fixative and frozen in liquid nitrogen.

\section{Histopathological examination}

Hematoxylin and eosin (H\&E) staining was performed. The fragment of the liver, distal ileum and colon were fixed in $4 \%$ neutral polyformaldehyde fixative, and following by steps: embedded in paraffin, cut into $5 \mu \mathrm{m}$ slices, deparaffinized, hydrated and stained. Thereafter, all tissue sections were examined by microscope.

\section{Liver sample preparation and quality control sample}

All of the tested liver samples were cut on the dry ice and weighted. $40 \mathrm{mg}$ of liver tissue was added $500 \mu \mathrm{L}$ of ultra-pure water to homogenate with a freeze tissue grinder at the frequency of 30 times/second, hold on 2 min, and repeat 3 times. $200 \mu \mathrm{L}$ of homogenate was moved to $1.5 \mathrm{EP}$ tubes and mixed with $800 \mathrm{uL}$ of methanol/acetonitrile $(1: 1, \mathrm{v} / \mathrm{v})$. The tubes were vortexed for $30 \mathrm{~s}$, and sonicate $10 \mathrm{~min}$ at $4{ }^{\circ} \mathrm{C}$ water bath. After incubating $1 \mathrm{~h}$ at $-20^{\circ} \mathrm{C}$, samples were centrifuged $15 \mathrm{~min}$ at $14500 \mathrm{rpm}$ at $4{ }^{\circ} \mathrm{C}$. The supernatant was evaporated to dryness use nitrogen blowing and reconstituted with $100 \mu \mathrm{L}$ acetonitrile/water $(1: 1, \mathrm{v} / \mathrm{v})$. Finally, the solution was filtered with a $0.22 \mu \mathrm{m}$ membrane and used for UPLC-HRMS analysis.

The quality control (QC) sample was used to assess the reproducibility and reliability of the UPLC-HRMS system and was prepared by mixing equal volumes of different individual liver samples. The pretreatment of QC sample is as same as the preparation of the above liver sample. The QC sample was inserted in each five samples during UPLC-HRMS analysis. The reproducibility and reliability of the method were assessed by coefficients of variation (CV\%) of 133 metabolites from QC samples.

\section{HRMS analysis}

The experiments were performed on UPLC system (Dionex UltiMate 3000) couple with orbitrap mass spectrometer (Thermo Fisher Scientific, Q-Exactive Focus). The UPLC-Orbitrap-MS/MS equipped with Xcalibur software (version 3.1) which used to instrument control, data acquisition and data analysis.

The chromatographic separation was performed on a C18 Hypersil Gold (100 mm $\times 2.1 \mathrm{~mm}, 1.9 \mu \mathrm{m}$, Thermo Scientific) column using an ultrapure water- $0.2 \%$ formic acid solution (eluent $A$ ) and acetonitrile (eluent $B$ ) as mobile phase at a flow rate of $0.2 \mathrm{~mL} / \mathrm{min}$. The gradient program was set as follows: $0-7 \mathrm{~min}, 5-50 \% \mathrm{~B} ; 7-8 \mathrm{~min}, 50-75 \% \mathrm{~B} ; 8-9 \mathrm{~min}$, $75-80 \% \mathrm{~B} ; 9-11 \mathrm{~min}, 80-90 \% \mathrm{~B} ; 11-15 \mathrm{~min}, 90-95 \% \mathrm{~B}$; equilibration time of $5 \mathrm{~min}$ at $5 \% \mathrm{~B}$, a total running time of 20 min. The column temperature $35^{\circ} \mathrm{C}$, and the injection volume was $2 \mu \mathrm{L}$.

The MS data were acquired using electrospray ionization (ESI) in positive ionization modes, spray voltage, $3.5 \mathrm{kV}(+3.5$ $\mathrm{kV}$ in ESI+); sheath gas (N2, > 95\%), 40 bar; auxiliary gas (N2, >95\%), 10 bar; heater temperature, $300{ }^{\circ} \mathrm{C}$; capillary temperature, $320^{\circ} \mathrm{C}$. MS Scanning mode: full MS scan ranged from m/z 70 to 1000 and the resolution was 35,000, insource collision-induced dissociation (in-source CID) was set at $0 \mathrm{eV}$. MS/MS scanning mode: Data-dependent ms 2 scan (dd-ms2) with the resolution was 17,000, loops was 3 and high collision induced dissociation (HCD) was set as stepped mode $(\mathrm{NCE}=10,30,50)$. 


\section{Data preprocessing and identification of metabolites}

Analytical instruments usually do not supply clean and visualized information of metabolites. Raw data must be processed to produce a workable data matrix thought a series pre-processing. In general, raw data preprocessing includes four basic modules, namely, namely, noise filtering and baseline correction, peak detection and deconvolution, alignment, and normalization. The experiments were performed on Compound Discoverer 2.1 (CD, Thermo Fisher Scientific) data analysis tool which is flexible and able to automate complete preprocessing according to presupposed parameters. Furthermore, the identification of metabolites uses accurate mass data, isotope pattern matching, and MS/MS spectral library searches. The CD has integrated various of tools to identify small molecule metabolites, including search mzCloud (online spectral library $>2$ million spectra), ChemSpider (chemical structure database with $>500$ data sources, 58 million structures), mzVault (local spectral libraries), and Masslist (local databases). Data preprocessing and metabolites identification were completed by one-stop, and the entire process is shown in Figure S1.

\section{Statistical analysis}

Chemometrics is one of the cornerstones of metabolomics because it can provide valuable and considerable knowledge from metabolites information. The statistic model was applied in almost metabolomics research. In this study, principal component analysis (PCA) and hierarchical cluster analysis (HCA) couple with orthogonal partial least-squares discriminant analysis (OPLS-DA) was performed to investigate the data of liver metabolites, and biomarkers were selected by S-plot of OPLS-DA. These methods mentioned above were performed on SIMCA-14.1 and Metaboanalyst 4.0 (http://www.metaboanalyst.ca). The metabolic network was analyzed and drawn using Metascape app which embedded in CytoScape3.7.1.

\section{Results}

\section{Establishment of DSS Induced IBD model in mice}

The IBD model induced by a dose of $2.5 \%$ DSS has been successfully established in our published work, and a series index includes weight, diarrhea score, bleeding score, and disease index were evaluated[12]. In addition, intestinal and liver tissue were used for histopathological analysis across H\&E staining as shown in Figure 1. Compared with control group (Figure 1, a and c), we can see distinctly in the DSS group that the villi of ileum (Figure 1b) were destroyed and the crypt was shortened, and the crypt of colon (Figure 1d) disappears completely. Furthermore, the result of liver morphology of DSS group (Figure 1f) shows hepatic cell swelling, cytoplasmic loosening, reticular and large numbers of hepatocyte apoptosis surrounding the vein. Those results indicated that inflammation occurs in the liver during the process of IBD.

\section{Validation of analytical performance}

We conducted PCA on all samples, and the results showed that the QC samples were tightly clustered in the middle of all samples in PCA score plot as shown in Figure 2d. The stability of the metabolomics analysis platform in the whole analysis process was demonstrated. The coefficients of variation of 133 metabolites from QC samples ranged from $2.74 \%$ to $17.83 \%$ (Supplementary Materials, Table S1). The results indicate that the method has good repeatability and reliability for metabolomics analysis.

\section{Identification of liver metabolites}


Liver samples were analyzed by UPLC-Orbitrap-MS/MS, and a very complicated raw data was obtained and displayed as total ion chromatogram (TIC) show in Figure S2. Effective mass spectra of peaks were extracted by CD software and matched with accurate mass data, isotope pattern and mass database. For example, taurocholic acid $[\mathrm{M}+\mathrm{H}]+\mathrm{at} \mathrm{m} / \mathrm{z}$ 516.2969 , and $\mathrm{m} / \mathrm{z} 517.3000, \mathrm{~m} / \mathrm{z} 518.2989$ of its isotope were found in the MS1(Figure S3a). Then, the MS/MS of taurocholic acid were matched mass databases such as mzCloud library and a score was given to indicate the similarity (Figure S3b). In this research, we choose the mzCloud score of peaks more than 75 and could match with ChemSpider, mzVault or Masslist. Finally, a total of 133 peaks were identified in each sample, and the Human Metabolome Database (HMDB) was used for further confirmation of metabolites. The detail information of metabolites was shown in Table S1. In addition, the chromatographic peak area of those metabolites has been normalized before multivariate analysis.

\section{Multivariate statistical analysis}

On the base of the qualitative and semiquantitative results of liver metabolites, chemometrics methods were used for digging deeper information of them. Firstly, PCA was performed to analyze the difference between DSS group and control group. The result of PCA (Figure 2a) revealed that metabolites in the different groups were separated into distinct clusters, and the first two principal components (PCs) of PCA accounted for greater than $50 \%$ of total variation. This implied that metabolism of liver was indeed changed due to IBD. The OPLS-DA model was established, subsequently, the result was shown as a three-dimensional (3D) scatter diagram in Figure 2b. Like the PCA result, OPLS-DA model shows clearly clusters of DSS group and control, and VIP calculated from OPLS-DA were listed in Table S1. In addition, 1000 times permutation tests (Figure 2c) were applied to estimate the reliability of the OPLS-DA mode and show Q2 and R2Y were 0.977 and 0.989 , respectively, indicating the model was effective.

In order to further illustrate the change of hepatic metabolism caused by IBD, two treatment groups (CA and GA) affiliated to DSS group and control to do statistical analysis. The result of PCA for these four groups shown in Figure 2d, dots of two treatment groups scattered in the middle between DSS and control group. The result suggested that some metabolites may arise reverse when the treatment (cecropin A or gentamicin) intervenes. In addition, metabolites which $p$ $<0.05$ were selected via one-way analysis of variance (one-way ANOVA), shown in Figure 2e, red dots mean $p<0.05$ and green dots mean $p>0.05$.

\section{Screening of metabolites}

Based on the results of multivariate statistical analysis, metabolites were screened. We selected 52 metabolites whose $p$ $<0.05$ using one-way ANOVA, and a heatmap analysis was performed to identify and differences and similarities among the four groups, as shown in Figure 3. Color changing of heatmap from dark red to deep blue indicated the downregulation and upregulation of the metabolites. Significant differences were observed between the DSS group (red) and control group (green), and a part of metabolites of CA (blue) and GA (light blue) group with a tendency to control group. The ANOVA post hoc analysis (DSS - control, CA -control and GA - control) was performed to reveal potential biomarkers, and the relative intensity of those metabolites in the four groups, and the semiquantitative results were listed in Table S2. Regarding the result of ANOVA post hoc analysis, there are 52 metabolites have significantly different $(p<$ 0.05) when DSS group compared to control, and CA and GA compared to control group, respectively, 29 and 19 compounds have no significant difference $(p>0.05)$.

The S-plot from OPLS-DA analysis was shown in Figure 4. In order to further screened metabolites, several values were used as restrictions include VIP > 1 in OPLS-DA, $p>0.05$ in ANOVA post hoc analysis (CA or GA compared to control) with least significant difference (LDS) and the absolute value of the correlation coefficient (Corr) $>0.80$ of S-plot. Finally, 20 compounds were selected by this limiting condition and were tagged use red dot in the S-plot. The detail of those 
metabolites was listed in Table 1 and the relative concentration changes of 20 metabolites in the 4 groups are shown in Figure 4 and Figure S4. Those metabolites were considered as potential biomarkers to distinguish between the DSS and control group, furthermore, which were reversed by the treatment of CA or GA.

\section{Metabolic pathway analysis}

In this research, we used the Metaboanalyst 4.0 platform to identify the metabolic pathway of liver which was influenced by IBD, and after treatment by CA and GA. All of the identified metabolites were searched and confirmed in HMDB, and only those have recorded pathways were analyzed. Figure 5 shown that the pathway analysis between the DSS and control group, DSS and CA group and the DSS and GA group, respectively. Obviously, there were extensive changes in the pathway of liver metabolism influenced by IBD. On the basis of an impact $>0.2$ and $\log (p)>5$, Figure 5 a shown the pathway with the significant impacts were taurine and hypotaurine metabolism, arachidonic acid metabolism, cysteine and methionine metabolism, aminoacyl-tRNA biosynthesis, glycine, serine and threonine metabolism, arginine and proline metabolism, glutathione metabolism, and alanine, aspartate and glutamate metabolism. Additionally, steroid hormone biosynthesis and tryptophan metabolism were also affected. Figure 5, b and c showed that the predominant pathway of liver metabolism by the treatment of CA and GA, and through its effects on series of amino acid metabolism, aminoacyl-tRNA biosynthesis, especially, an obviously regulating effect on taurine and hypotaurine metabolism, glutathione metabolism, and arachidonic acid metabolism.

The metabolism network analysis related to the three treatment groups, as shown in Figure 6. There were 21 downregulated compounds and 22 upregulated compounds in the metabolic network of DSS group (Figure 6a). In the GA group (Figure 6b), 11 compounds were downregulated and 27 compounds were upregulated, whereas only 9 compounds were downregulated and 16 upregulated compounds in CA group (Figure 6c).

\section{Discussion}

\section{DSS induced IBD cause perturbations in liver metabolism}

Regarding the data presented in Figure 5a and Figure 6a, it could be concluded that the changing of liver metabolism caused by DSS induced IBD may be primarily through these several interferential aspects: (a) bile acid metabolism disorder (taurine, taurocholate, hypotaurine). (b) arachidonic acid metabolism disorder. (c) amino acid and protein disorder (aspartic, glutathione, glutamate acid, proline acid). (d) steroid hormone biosynthesis (cortisol, cortisone, corticosterone, 11-Dehydrocorticosterone).

Bile acids were liver-derived cholesterol derivates that control digestion and modulate lipid metabolism[20]. Taurocholate is one primarily compound metabolite in bile acid metabolism and is synthesized from taurine and hypotaurine. As it is shown in Figure 4, Figure 5a and Figure 6a shown, the level of hypotaurine, taurine, taurocholate was raised in DSS group. The result may imply that excessive bile acid was generated in the liver. Many published studies suggested the importance of appropriately maintaining bile acids homeostasis to liver metabolism, and bile acids overload will cause inflammation and impaired liver[21-23]. Furthermore, recent research showed that the gut microbiota has a fundamental role in many disorders within and beyond the gastrointestinal tract[24, 25]. Gut microbiota affects intestinal signaling and enterohepatic circulation of bile acids via a "liver-microbiome axis" $[6,26]$. So, in this research, the bile acids metabolism disorder may be caused by the disturbers of gut microbiota because of IBD.

Compared with the control group, arachidonic acid significant decreased in DSS group, shown in Figure 4 and Figure 6 a. Arachidonic acid is a polyunsaturated fatty acid essential for normal health and is a component of the biological cell membrane, which can maintain the normal permeability and flexibility of cells[27]. Noteworthiness, during inflammation, 
arachidonic acid reacts with enzymes to form prostaglandins, leukotrienes, and thromboxanes which were considered predominantly as proinflammatory molecules[28]. Therefore, arachidonic acid down-regulation may be caused by inflammation in the liver.

The liver is the primary metabolic organs for amino acids and proteins. Evidently, the DSS group was altered in amino acid and protein metabolism because there were 11 amino acid-related compounds that were regulated in 5 metabolic pathways of the liver metabolism, as shown in Figure 5a and Figure 6a. Especially, pyroglutamic acid, glutamic acid, and glutathione are involved in glutathione metabolism and were down-regulated in DSS group. The glutathione pathway is a key hepatic defense mechanism and deactivates reactive metabolites before they have the chance to damage cellular proteins[29]. In addition, we found that tryptophan metabolism was influenced significantly by IBD in our research, shown in Figure 5a. Tryptophan is an essential amino acid involved in many biological processes and its metabolites play a crucial role in regulation of immunity[30]. There are three major pathways of tryptophan metabolism, including serotonin pathway, kynurenine pathway and indole pathway[25]. In our research, melatonin, kynurenine, indole-3-acetate which were key metabolites in these three pathways of tryptophan, have been detected and observed kynurenine significantly upregulated. Recently published studies show gut microbiota can effect on host physiology and pathology by interfering with tryptophan metabolism[31], such as patients treated with interferon for hepatitis, massively tryptophan is used to generated kynurenine inducing insufficient tryptophan and serotonin in brain, subsequently leading to depression[32]. Therefore, in the process of IBD, the disorder of tryptophan metabolism in liver may be caused by the intervention mechanism of gut microbiota on tryptophan metabolism.

IBD also caused the disarray of steroid hormone biosynthesis. Glucocorticoids are primary steroid hormones including cortisol and cortisone, which are related factors indicating stress and pain, and play a decisive role in metabolism, maintaining energy balance and animal survival in adversity[33]. An appropriate stress response is conducive to the body's short-term adaptation to the environment, however, long-term stress or strong stimulation, high level of glucocorticoids is bounded to cause energy metabolism and hormone secretion disorder, thereby affecting health[34]. Published research found that excessive cortisol secretion will promote the catabolism of proteins in extrahepatic tissues, resulting in negative nitrogen balance[35]. In our study, IBD kept mice in long-term stress and promoted excessive glucocorticoid secretion (cortisol and cortisone as show Figure 4 and Figure S4), which may induce liver injury.

\section{Cecropin A and gentamicin reversed the metabolism changes in liver of IBD-induced}

The gut microflora is an important factor in regulating gastrointestinal homeostasis, affecting the immune system and host metabolism[36]. In recent studies, gut microflora has been shown to be closely related to the development of hepatopathy [37]. In our previous studies, we found cecropin A and gentamicin could alleviate IBD across regulating microbiota population, and cecropin A has better effectiveness compared to gentamicin[12]. Figure $6, b$ and $c$ were liver metabolism network analysis of CA and GA to control group. CA clearly reversed bile acid metabolism, amino acid and protein and steroid hormone biosynthesis disarray caused by IBD. The upregulation of taurine, taurocholate, hypotaurine, cortisol, cortisone, corticosterone, 11-Dehydrocorticosterone in the DSS group disappeared in the CA group. The glutathione and metabolites of tryptophan metabolism returned to normal levels. Compared to CA, the reversal ability of GA group was weak, only glutathione and arachidonic acid were reversed. The possible reasons of these results were that cecropin A can improve the abundance of beneficial bacteria and reduce the adhesion of harmful bacteria to cells[38], which may benefit for the intestinal epithelium recovery and regulating gut microbiota, thereby alleviate IBD and liver metabolism disturbance. On the other hand, cecropin A and gentamicin showed different effects on their microbiota population, which may lead to different degrees of recovery from intestinal injury. 


\section{Conclusion}

This study concentrates on reveling metabolic profiles of liver in mice with DSS-induced IBD. A total of 133 compounds were obtained by UPLC-HRMS combined with highly effective non-targeted qualitative tools, and 20 metabolic markers were screened by chemometrics. Metabolic pathway analysis and metabolites network analysis indicated that bile acid metabolism, arachidonic acid metabolism, amino acid and protein metabolism, steroid hormone biosynthesis have been changed in liver caused by IBD. In addition, CA and GA treatments assisted in verifying the correlation between IBD and liver metabolism. We hope our efforts may provide references in the study of IBD associated with molecular mechanisms in liver.

\section{Abbreviations}

IBD: Inflammatory bowel disease; DSS: dextran sulfate sodium; CD: Crohn's disease; UC: ulcerative colitis; EIMs: extraintestinal manifestations; UPLC-HRMS: ultra-high performance liquid chromatography coupled with high-resolution mass spectrometry; LC-MS: liquid chromatography mass spectrometry; GC-MS: gas chromatography mass spectrometry; CA: Cecropin A; GA: Gentamicin; H\&E: Hematoxylin and eosin; QC: quality control; CV: coefficients of variation; ESI: electrospray ionization; CID: collision-induced dissociation; HCD: high collision induced dissociation; PCA: principal component analysis; HCA: hierarchical cluster analysis; OPLS-DA: orthogonal partial least-squares discriminant analysis; TIC: total ion chromatogram; HMDB: Human Metabolome Database; LDS: least significant difference.

\section{Declarations}

\section{Ethics approval and consent to participate}

The experimental design and procedures in this study were reviewed and approved by the Animal Care and Use Committee of the Institute of Subtropical Agriculture, Chinese Academy of Science (No.ISA-2018-035). The National Centre for the Replacement, Refinement and Reduction of Animals in Research (NC3Rs) handling guidelines were also followed within this study. A An animal research: reporting of in-vivo experiments (ARRIVE) guidelines checklist for working with animals can be found in Additional file 1.

\section{Consent for publication}

The authors have been informed and agreed to publish the article in the journal.

\section{Competing interests}

Not applicable

\section{Availability of data and materials}

The datasets used and/or analyzed during the current study are available from the corresponding author on reasonable request.

\section{Founding}


This project was supported by the National Key R\&D Program of China (Grant No. 2018YFD0500600), National Natural Science Foundation of China for support of the projects (Grant No.31790411), Natural Science Foundation of Guangdong Province, China (Grant No. 2017A030310410), Innovation Team Project in Universities of Guangdong Province (2017KCXTD002). This project was approved by the university's review board. Baichuan Deng participated in the design of the experiment and revised the manuscript.

\section{Authors' contributions}

ZX and ZZ devised the research concept. JD, LY and BD designed the experimental protocol. FZ, HL and XN performed the experiments. ZX and BD acquired the data and performed the data analysis. ZX and LY were involved in interpreting the result. ZX drafted the original manuscript. All authors were involved in revising the manuscript and approving the final version.

\section{Acknowledgements}

Not applicable.

\section{References}

1.Franzosa EA, Sirota-Madi A, Avila-Pacheco J, Fornelos N, Haiser HJ, Reinker S, Vatanen T, Hall AB, Mallick H, Mclver LJ et al: Gut microbiome structure and metabolic activity in inflammatory bowel disease. Nature microbiology 2019, 4(2):293-305.

2.Abraham C, Cho JH: MECHANISMS OF DISEASE Inflammatory Bowel Disease. N Engl J Med 2009, 361(21):2066-2078.

3.Annese V: A Review of Extraintestinal Manifestations and Complications of Inflammatory Bowel Disease. Saudi journal of medicine \& medical sciences 2019, 7(2):66-73.

4.Uko V, Thangada S, Radhakrishnan K: Liver disorders in inflammatory bowel disease. Gastroenterology research and practice 2012, 2012:642923.

5.Mendes FD, Levy C, Enders FB, Loftus EV, Jr., Angulo P, Lindor KD: Abnormal hepatic biochemistries in patients with inflammatory bowel disease. The American journal of gastroenterology 2007, 102(2):344-350.

6.Adolph TE, Grander C, Moschen AR, Tilg H: Liver-Microbiome Axis in Health and Disease. Trends in Immunology 2018, 39(9):712-723.

7.Mazagova M, Wang LR, Anfora AT, Wissmueller M, Lesley SA, Miyamoto Y, Eckmann L, Dhungana S, Pathmasiri W, Sumner S et al: Commensal microbiota is hepatoprotective and prevents liver fibrosis in mice. Faseb J 2015, 29(3):1043-1055.

8.Fox JG, Feng Y, Theve EJ, Raczynski AR, Fiala JL, Doernte AL, Williams M, McFaline JL, Essigmann JM, Schauer DB et al: Gut microbes define liver cancer risk in mice exposed to chemical and viral transgenic hepatocarcinogens. Gut 2010, 59(1):88-97.

9.Sheehan D, Shanahan F: The Gut Microbiota in Inflammatory Bowel Disease. Gastroenterology clinics of North America 2017, 46(1):143-154. 
10.Ledder O: Antibiotics in inflammatory bowel diseases: do we know what we're doing? Translational pediatrics 2019, $8(1): 42-55$.

11.laniro G, Tilg H, Gasbarrini A: Antibiotics as deep modulators of gut microbiota: between good and evil. Gut 2016, 65(11):1906-1915.

12.Zhai ZY, Zhang F, Cao RH, Ni XJ, Xin ZQ, Deng JP, Wu GY, Ren WK, Yin YL, Deng BC: Cecropin A Alleviates Inflammation Through Modulating the Gut Microbiota of C57BL/6 Mice With DSS-Induced IBD. Front Microbiol $2019,10$.

13.Zhang A, Sun H, Wang P, Han Y, Wang X: Modern analytical techniques in metabolomics analysis. The Analyst 2012, 137(2):293-300.

14.Kluger B, BueschI C, Neumann N, Stuckler R, Doppler M, Chassy AW, Waterhouse AL, Rechthaler J, Kampleitner N, Thallinger GG et al: Untargeted profiling of tracer-derived metabolites using stable isotopic labeling and fast polarityswitching LC-ESI-HRMS. Analytical chemistry 2014, 86(23):11533-11537.

15.Ren D, Ran L, Yang C, Xu M, Yi L: Integrated strategy for identifying minor components in complex samples combining mass defect, diagnostic ions and neutral loss information based on ultra-performance liquid chromatography-high resolution mass spectrometry platform: Folium Artemisiae Argyi as a case study. Journal of chromatography A 2018, 1550:35-44.

16.Yi L, Dong N, Yun Y, Deng B, Ren D, Liu S, Liang Y: Chemometric methods in data processing of mass spectrometrybased metabolomics: A review. Analytica chimica acta 2016, 914:17-34.

17.Tautenhahn R, Patti GJ, Rinehart D, Siuzdak G: XCMS Online: a web-based platform to process untargeted metabolomic data. Analytical chemistry 2012, 84(11):5035-5039.

18.Pluskal T, Castillo S, Villar-Briones A, Oresic M: MZmine 2: modular framework for processing, visualizing, and analyzing mass spectrometry-based molecular profile data. BMC bioinformatics 2010, 11:395.

19.Ma SS, Chen L, Li J, Wang ZY, Xin ZQ, Zhang Y, Ren DB, Yi LZ: Characterization and authentication of Acori Tatarinowii Rhizoma and its adulterants by UPLC-Orbitrap-MS/MS chromatographic fingerprints, elements profiles and chemometric methods. Microchem J 2019, 144:285-295.

20.Chen W, Wei Y, Xiong A, Li Y, Guan H, Wang Q, Miao Q, Bian Z, Xiao X, Lian M: Comprehensive Analysis of Serum and Fecal Bile Acid Profiles and Interaction with Gut Microbiota in Primary Biliary Cholangitis. Clinical Reviews in Allergy \& Immunology 2019:1-14.

21.Pean N, Doignon I, Garcin I, Besnard A, Julien B, Liu B, Branchereau S, Spraul A, Guettier C, Humbert L et al: The receptor TGR5 protects the liver from bile acid overload during liver regeneration in mice. Hepatology 2013, 58(4):14511460.

22.Huang WD, Ma K, Zhang J, Qatanani M, Cuvillier J, Liu J, Dong BN, Huang XF, Moore DD: Nuclear receptor-dependent bile acid signaling is required for normal liver regeneration. Science 2006, 312(5771):233-236.

23.Liu HX, Keane R, Sheng L, Wan YJ: Implications of microbiota and bile acid in liver injury and regeneration. Journal of hepatology 2015, 63(6):1502-1510.

24.Costea PI, Hildebrand F, Manimozhiyan A, Backhed F, Blaser MJ, Bushman FD, de Vos WM, Ehrlich SD, Fraser CM, Hattori M et al: Enterotypes in the landscape of gut microbial community composition. Nature microbiology 2018, 3(1). 
25.Lynch SV, Pedersen O: The Human Intestinal Microbiome in Health and Disease. N Engl J Med 2016, 375(24):23692379 .

26.Wahlstrom A, Sayin SI, Marschall HU, Backhed F: Intestinal Crosstalk between Bile Acids and Microbiota and Its Impact on Host Metabolism. Cell metabolism 2016, 24(1):41-50.

27.Tallima H, El Ridi R: Arachidonic acid: Physiological roles and potential health benefits - A review. Journal of advanced research 2018, 11:33-41.

28.Poorani R, Bhatt AN, Dwarakanath BS, Das UN: COX-2, aspirin and metabolism of arachidonic, eicosapentaenoic and docosahexaenoic acids and their physiological and clinical significance. Eur J Pharmacol 2016, 785:116-132.

29.Gould RL, Zhou Y, Yakaitis CL, Love K, Reeves J, Kong WQ, Coe E, Xiao YF, Pazdro R: Heritability of the aged glutathione phenotype is dependent on tissue of origin. Mamm Genome 2018, 29(9-10):619-631.

30.Roager HM, Licht TR: Microbial tryptophan catabolites in health and disease. Nature communications 2018, 9(1):3294.

31.Agus A, Planchais J, Sokol H: Gut Microbiota Regulation of Tryptophan Metabolism in Health and Disease. Cell host \& microbe 2018, 23(6):716-724.

32. Chaves Filho AJM, Lima CNC, Vasconcelos SMM, de Lucena DF, Maes M, Macedo D: IDO chronic immune activation and tryptophan metabolic pathway: A potential pathophysiological link between depression and obesity. Prog Neuropsychopharmacol Biol Psychiatry 2017, 80(Pt C):S0278584616303451.

33.Magomedova L, Cummins CL: Glucocorticoids and Metabolic Control. 2015, 233:73-93.

34.Dedovic K, Duchesne A, Andrews J, Engert V, Pruessner JC: The brain and the stress axis: The neural correlates of cortisol regulation in response to stress. Neuroimage 2009, 47(3):864-871.

35.Rinard GA, Okuno G, Haynes RC, Jr.: Stimulation of gluconeogenesis in rat liver slices by epinephrine and glucocorticoids. Endocrinology 1969, 84(3):622-631.

36.Sunkara T, Rawla P, Ofosu A, Gaduputi V: Fecal microbiota transplant - a new frontier in inflammatory bowel disease. Journal of inflammation research 2018, 11:321-328.

37.Jiang WW, Wu N, Wang XM, Chi YJ, Zhang YY, Qiu XY, Hu Y, Li J, Liu YL: Dysbiosis gut microbiota associated with inflammation and impaired mucosal immune function in intestine of humans with non-alcoholic fatty liver disease. Sci Rep-Uk 2015, 5.

38.Zhai ZY, Ni XJ, Jin CL, Ren WK, Li J, Deng JP, Deng BC, Yin YL: Cecropin A Modulates Tight Junction-Related Protein Expression and Enhances the Barrier Function of Porcine Intestinal Epithelial Cells by Suppressing the MEK/ERK Pathway. Int J Mol Sci 2018, 19(7).

\section{Tables}

Table 1. The 20 significant metabolites in liver of control, DSS, CA and GA group 


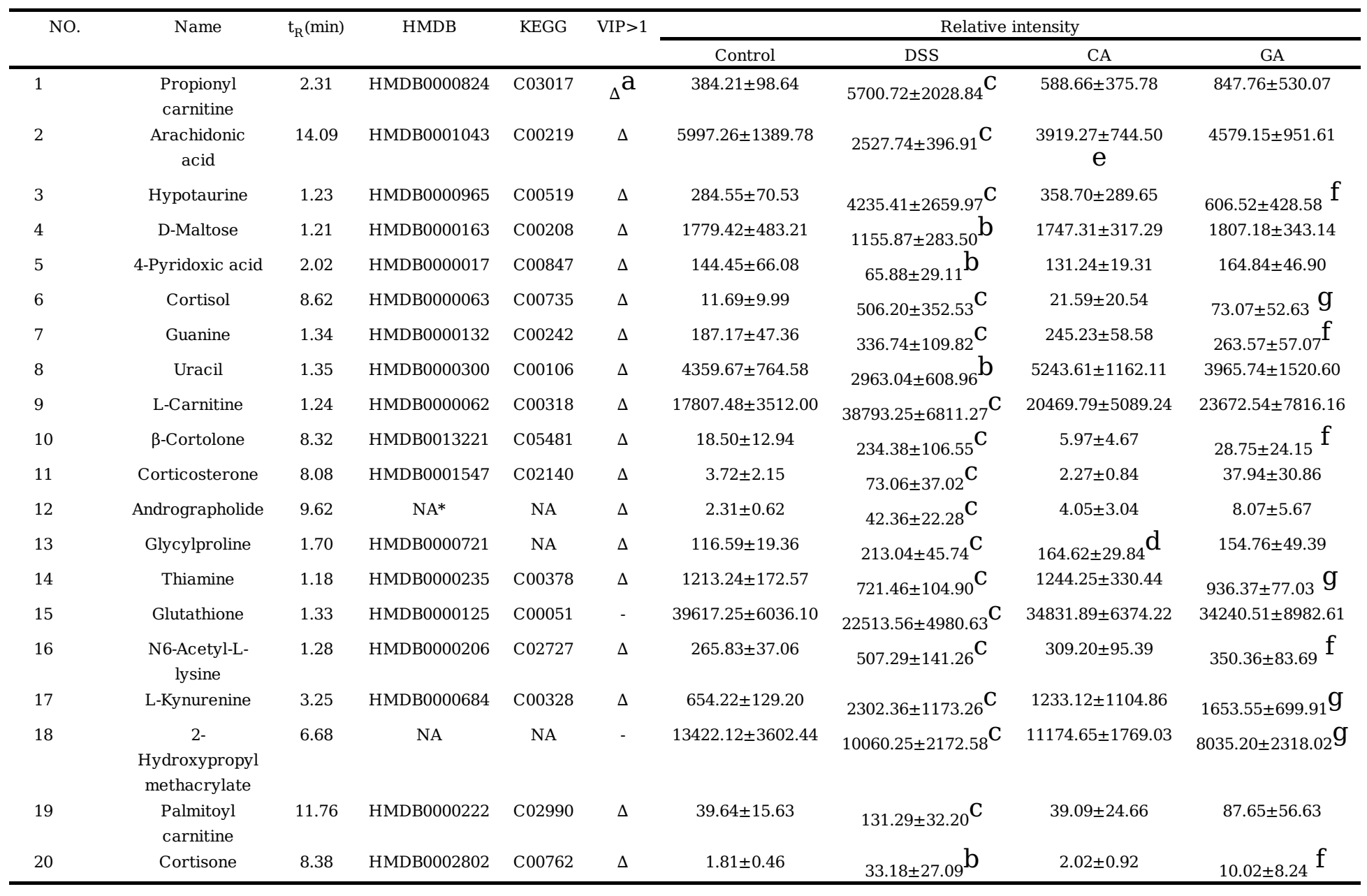

*NA means not available. ${ }^{\mathrm{a}} \Delta$ means VIP $>1$ in OPLS-DA. ${ }^{\mathrm{b}} \mathrm{DSS}$ group compared with control group, $p<0.05 .{ }^{\mathrm{c}} \mathrm{DSS}$ group compared with control group, $p<0.01 .{ }^{\mathrm{d}} \mathrm{CA}$ group compared with control group, $p<0.05 .{ }^{e} \mathrm{CA}$ group compared with control group, $p<0.01 .{ }^{\mathrm{f}} \mathrm{GA}$ group compared with control group, $p<0.05 .{ }^{\mathrm{g}} \mathrm{GA}$ group compared with control group, $p<0.01$.

\section{Figures}



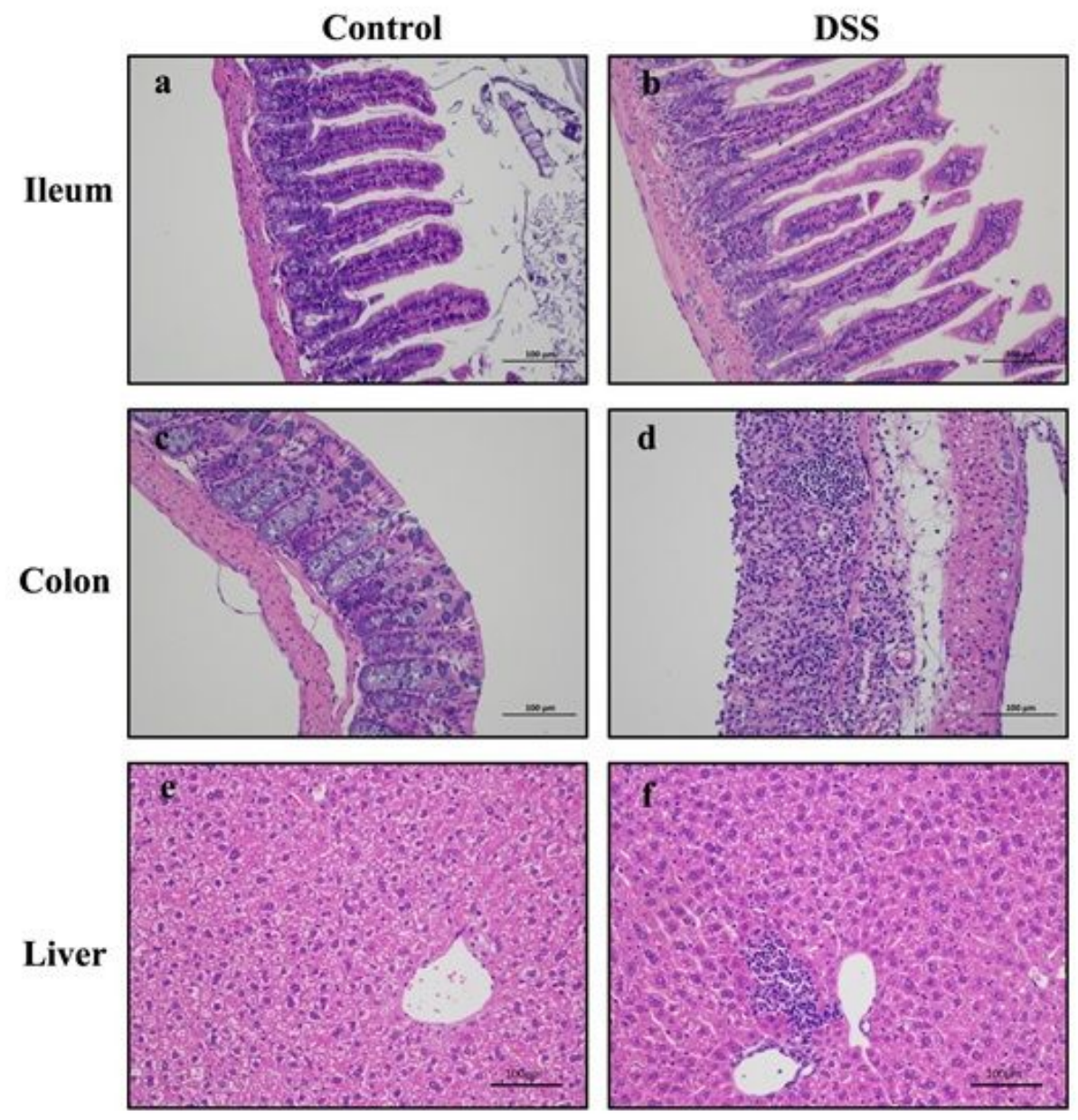

\section{Figure 1}

Histopathological results of ileum, colon, and liver stained with H\&E of control group ( $a, c$ and e) and DSS group (b, $d$ and f)
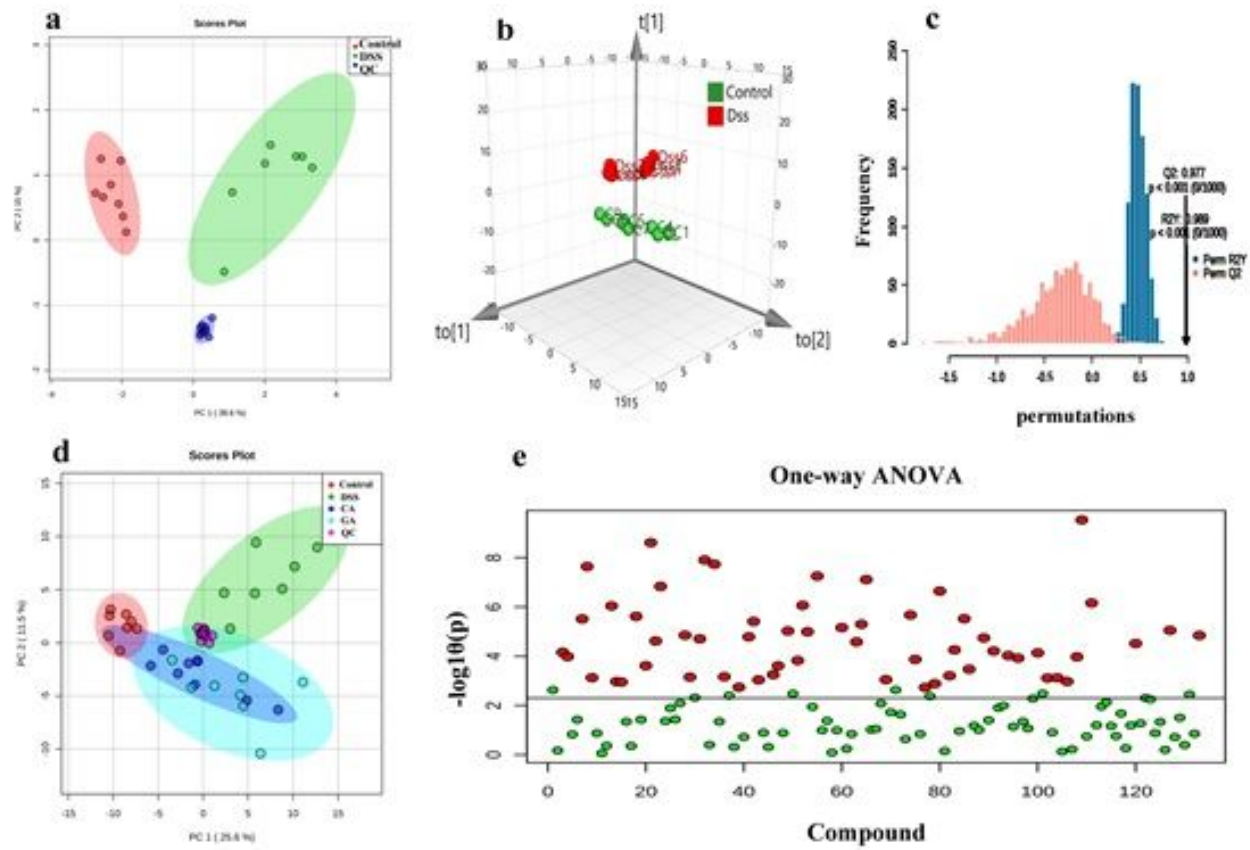

Figure 2 
Multivariate statistical analysis of the four group: control group, DSS group, CA group and GA group. (a) principal component analysis (PCA) of DSS group, control group and QC. (b) Orthogonal partial least-squares discriminant analysis (OPLS-DA) between DSS group and control group. (c) Permutation test to evaluated OPLS-DA. (d) PCA of DSS group, control group, cecropin A group (CA), Gentamicin group (GA) and QC. (e) One-way analysis of variance of metabolites in those four groups, red dots represent metabolites with $p<0.05$ and green dot represent $p>0.05$

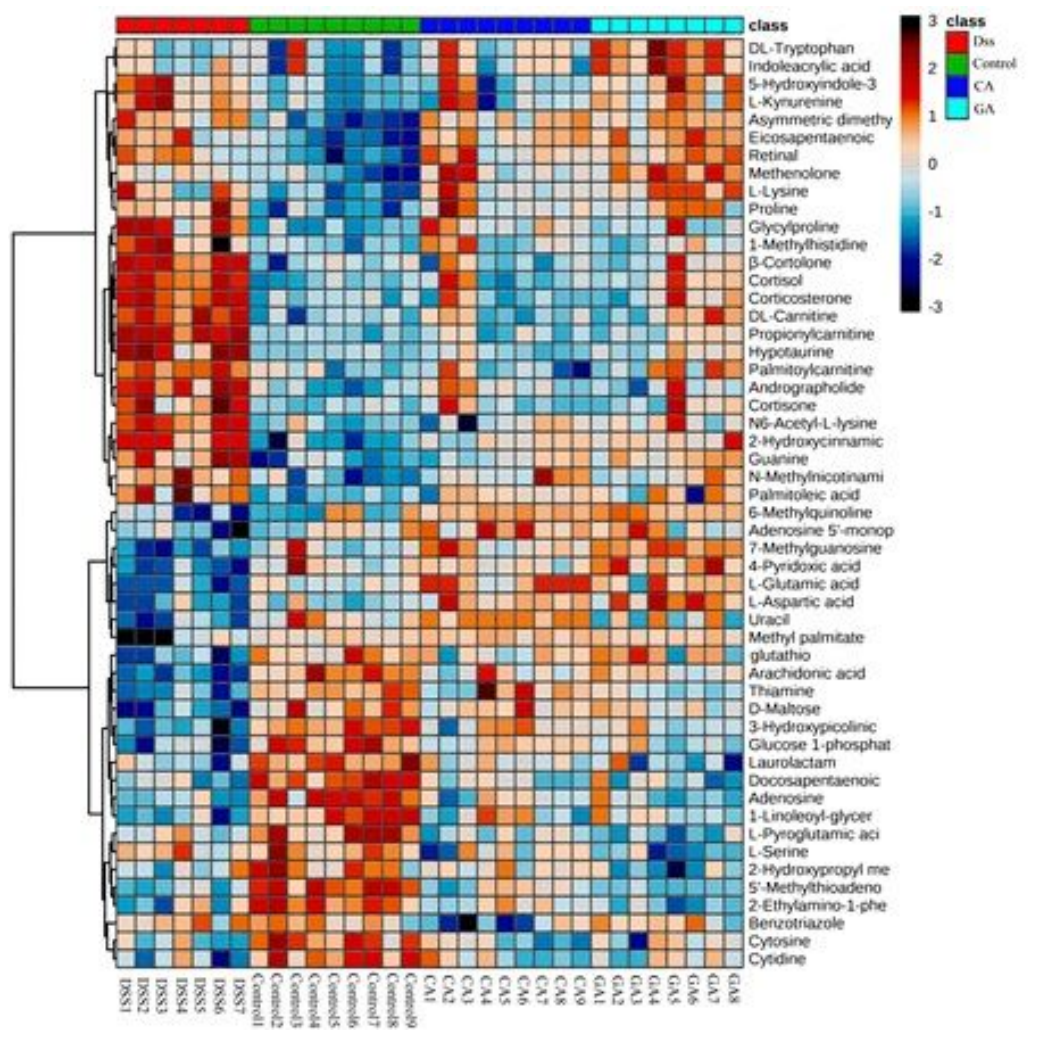

Figure 3

Heat map of the cluster analysis of the 52 metabolites with a $p<0.05$ calculated by one-way ANOVA. DSS group marked with red, control group marked with green, CA group marked with blue and GA group marked with light blue
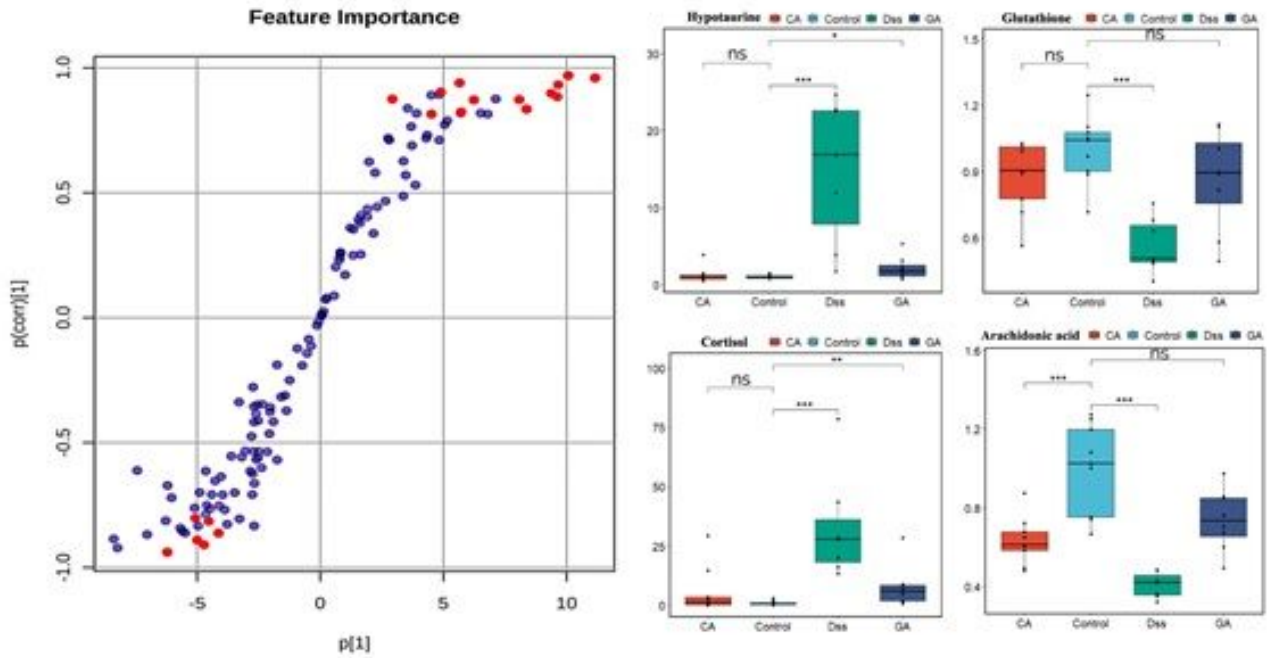

\section{Figure 4}

S-plot obtained from the OPLS-DA analysis of DSS group and control group. We used red dots to mark potential biomarkers which were $p<0.05$ in the one-way ANOVA, $p>0.05$ in ANOVA post hoc analysis (CA or GA compared to 
control) with LSD, VIP $>1$ in OPLS-DA and the absolute value of the correlation coefficient (Corr) $>0.80$ of S-plot. Box-plot shows the concentration changes of the potential biomarkers in the four groups $\left({ }^{*} p<0.05, * \star p<0.01,{ }^{*}{ }^{*} p<0.001, n s\right.$ means no significantly). Due to the limitation of space, only a part of them was listed, the rest can be found in supplementary materials (Figure S4)
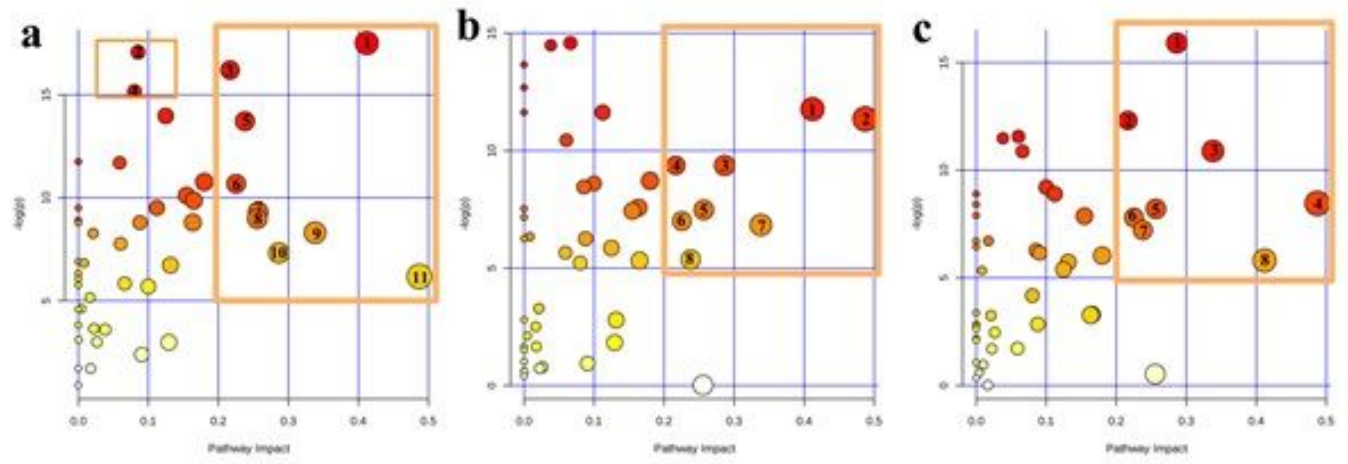

\section{Figure 5}

Metabolism pathway analysis of the four groups. The x-axis represents the pathway impact, and the $y$-axis represents the pathway enrichment. The critical pathways are marked with yellow boxes. (a) Analysis between the DSS group and control group (DSS vs Control). 1-taurine and hypotaurine metabolism, 2-steroid hormone biosynthesis, 3-arachidonic acid metabolism, 4-tryptophan metabolism, 5-cysteine and methionine metabolism, 6-aminoacyl-tRNA biosynthesis, 7glycine, serine and threonine metabolism, 8-retinol metabolism, 9- glutathione metabolism, 10- arginine and proline metabolism, 11-alanine, aspartate and glutamate metabolism. (b) Analysis between the CA group and DSS group (DSS vs CA). 1-taurine and hypotaurine metabolism, 2-alanine, aspartate and glutamate metabolism, 3-glutathione metabolism, 4-arachidonic acid metabolism, 5-glycine, serine and threonine metabolism, 6-aminoacyl-tRNA biosynthesis, 7-arginine and proline metabolism, 8-cysteine and methionine metabolism. (c) Analysis between the GA group and DSS group (DSS vs GA). 1-glutathione metabolism, 2-arachidonic acid metabolism, 3-arginine and proline metabolism, 4alanine, aspartate and glutamate metabolism, 5-glycine, serine and threonine metabolism, 6-aminoacyl-tRNA biosynthesis, 7-taurine and hypotaurine metabolism, 8-cysteine and methionine metabolism

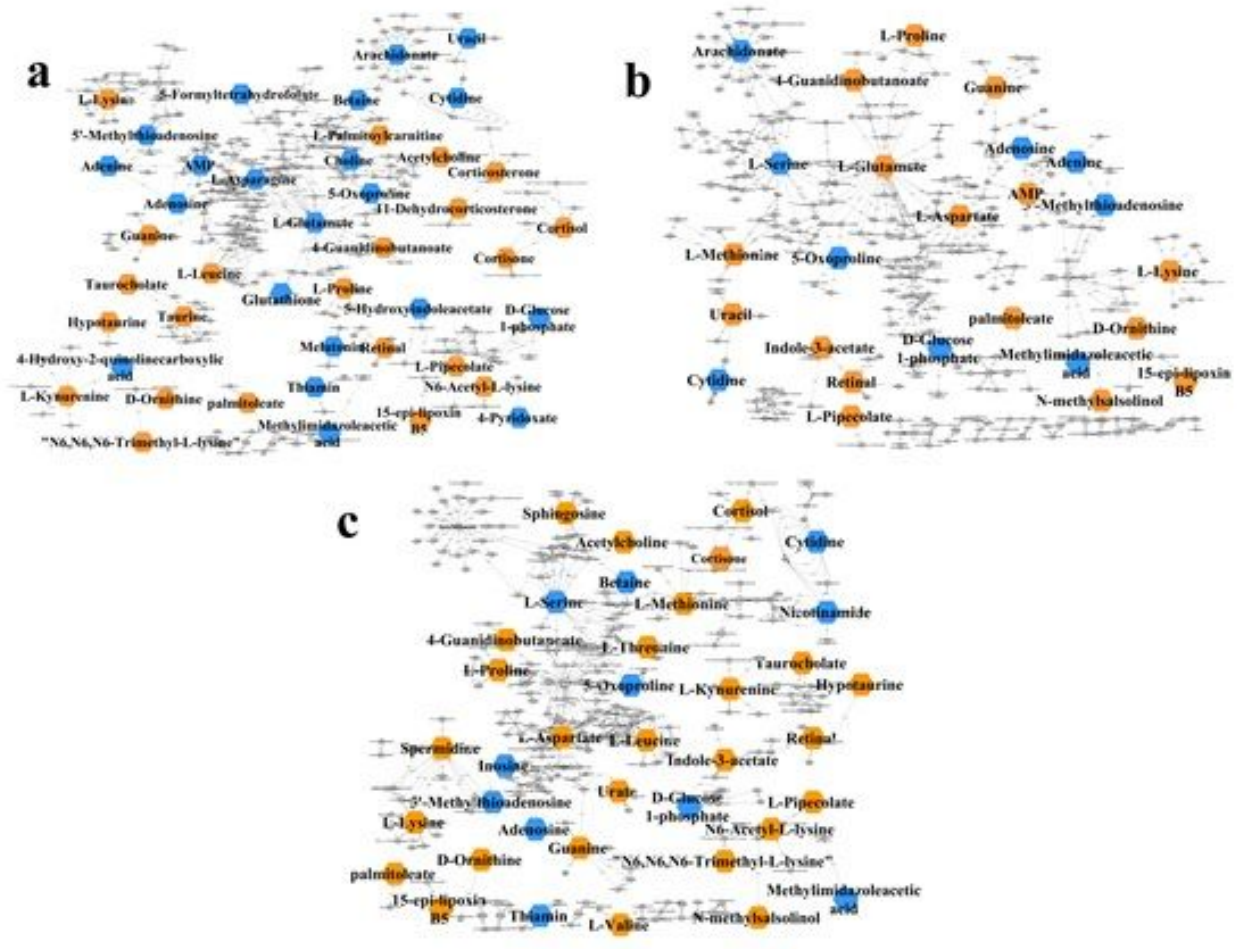

Page $16 / 17$ 


\section{Figure 6}

Metabolism network analysis of three treatment groups: (a) DSS group vs control group. (b) CA group vs control group. (c) GA group vs control group. Blue was used to indicate downregulated metabolites, and red was used to denote upregulated metabolites

\section{Supplementary Files}

This is a list of supplementary files associated with this preprint. Click to download.

- Additionalfile1ARRIVEchecklist.docx

- MetabolicprofilesofliverSI.docx 